\title{
Radiation-pressure-driven dust waves inside bursting interstellar bubbles
}

\author{
B. B. Ochsendorf ${ }^{1}$, S. Verdolini ${ }^{1}$, N. L. J. Cox ${ }^{2}$, O. Berné ${ }^{3}$, L. Kaper ${ }^{4}$, and A. G. G. M. Tielens ${ }^{1}$ \\ ${ }^{1}$ Leiden Observatory, Leiden University, PO Box 9513, 2300 RA, The Netherlands \\ e-mail: ochsendorfestrw. leidenuniv.nl \\ 2 Instituut voor Sterrenkunde, K.U. Leuven, Celestijnenlaan 200D, bus 2401, 3001 Leuven, Belgium \\ 3 Université de Toulouse, UPS-OMP, IRAP, 31028 Toulouse, France \\ ${ }^{4}$ Sterrenkundig Instituut Anton Pannekoek, University of Amsterdam, Science Park 904, PO Box 94249, 1090 GE Amsterdam, \\ The Netherlands
}

Received 30 January 2014 / Accepted 10 April 2014

\begin{abstract}
Massive stars drive the evolution of the interstellar medium through their radiative and mechanical energy input. After their birth, they form "bubbles" of hot gas surrounded by a dense shell. Traditionally, the formation of bubbles is explained through the input of a powerful stellar wind, even though direct evidence supporting this scenario is lacking. Here we explore the possibility that interstellar bubbles seen by the Spitzer- and Herschel space telescopes, blown by stars with $\log \left(L / L_{\odot}\right) \lesssim 5.2$, form and expand because of the thermal pressure that accompanies the ionization of the surrounding gas. We show that density gradients in the natal cloud or a puncture in the swept-up shell lead to an ionized gas flow through the bubble into the general interstellar medium, which is traced by a dust wave near the star, which demonstrates the importance of radiation pressure during this phase. Dust waves provide a natural explanation for the presence of dust inside HII bubbles, offer a novel method to study dust in H II regions and provide direct evidence that bubbles are relieving their pressure into the interstellar medium through a champagne flow, acting as a probe of the radiative interaction of a massive star with its surroundings. We explore a parameter space connecting the ambient density, the ionizing source luminosity, and the position of the dust wave, while using the well studied H II bubbles RCW 120 and RCW 82 as benchmarks of our model. Finally, we briefly examine the implications of our study for the environments of super star clusters formed in ultraluminous infrared galaxies, merging galaxies, and the early Universe, which occur in very luminous and dense environments and where radiation pressure is expected to dominate the dynamical evolution.
\end{abstract}

Key words. ISM: bubbles - HII regions - infrared: ISM - hydrodynamics - ISM: kinematics and dynamics - dust, extinction

\section{Introduction}

The morphological appearance of mid-infrared (MIR) bubbles in the disk of the Galaxy is traditionally explained by the interaction of a stellar wind with the surrounding gas (Weaver et al. 1977). In this scenario, stellar winds from massive stars drive shock waves in their surroundings, which sweep up the ambient gas and produce a cavity filled with hot $\left(T \sim 10^{7} \mathrm{~K}\right)$, tenuous $\left(n_{\mathrm{H}} \sim 0.01 \mathrm{~cm}^{-3}\right)$, collisionally ionized gas surrounded by a dense $\left(n_{\mathrm{H}} \sim 10^{5} \mathrm{~cm}^{-3}\right)$ shell. Assisted by volunteers from the general public, some 5000 bubbles have now been identified in the Galactic plane (Churchwell et al. 2006; Simpson et al. 2012).

The evolution of expanding H II regions has been addressed numerically in several recent papers, investigating the influence of the initial cloud structure on bubble expansion (i.e., Walch et al. 2013) and the effect of stellar winds (Dale et al. 2013). In addition, a theoretical study including stellar winds is described in Raga et al. (2012). Still, observations challenge our understanding on wind-blown bubbles (WBB). First, detection of the hot gas through X-ray emission has proven to be elusive for bubbles powered by a handful of OB stars. Diffuse $\mathrm{X}$-ray emission has only been detected towards sources with extreme mass-loss (Wolf-Rayet bubbles; Toalá et al. 2012; Toalá \& Guerrero 2013), extreme ionizing power (M17 and the Rosette nebula; Townsley et al. 2003), and superbubbles created by interior supernova remnants (Chu \& Mac Low 1990). Explanations for the lack of X-ray detections inside the vast majority of the bubbles include mass loading, thermal conduction, and leakage of the hot gas from the bubble (Harper-Clark \& Murray 2009; Arthur 2012). Second, recent observations show that winds from O stars with $\log \left(L / L_{\odot}\right) \lesssim 5.2$ (Martins et al. 2005b; Marcolino et al. 2009) may be less powerful than long thought (the weakwind problem; Puls et al. 2008; Najarro et al. 2011), which in its turn is challenged by recent X-ray observations of a stellar wind described in Huenemoerder et al. (2012). Third, dust grains are expected to evacuate the interiors of WBBs on short timescales $\left(\sim 10^{5} \mathrm{yr}\right)$ by either acceleration or sputtering due to friction with high-velocity gas $\left(\sim 2000 \mathrm{~km} \mathrm{~s}^{-1}\right)$ from the stellar wind (Everett \& Churchwell 2010). Yet, high-resolution infrared observations show that $\mathrm{H}$ II bubbles include a significant amount of dust in their interiors (Deharveng et al. 2010; Martins et al. 2010; Anderson et al. 2012). The evaporation of small, dense cloudlets could resupply the hot gas with a new generation of dust grains (Everett \& Churchwell 2010). While this mechanism can explain the presence of dust inside H II bubbles, it does not produce in a natural way the morphology of the (mid-)IR radiation: arc-shaped and peaking close to the ionizing source.

Driven by the difficulties in connection with the model of WBBs outlined above, we have explored the possibilities of H II bubble formation by thermal pressure (Spitzer 1978) instead of a stellar wind. We present the observations and the 
Table 1. Surveys used in this study.

\begin{tabular}{lcl}
\hline \hline Survey & Passband & Reference \\
\hline SuperCOSMOS & $0.656 \mu \mathrm{m}$ & Parker \& Phillipps (1998) \\
Spitzer/GLIMPSE & $8 \mu \mathrm{m}$ & Benjamin et al. (2003) \\
Spitzer/MIPSGAL & $24 \mu \mathrm{m}$ & Carey et al. (2009) \\
Herschel/Hi-Gal & $250 \mu \mathrm{m}$ & Molinari et al. (2010) \\
NVSS & $1.4 \mathrm{GHz}$ & Condon et al. (1998) \\
SUMSS & $843 \mathrm{MHz}$ & Bock et al. (1999) \\
\hline
\end{tabular}

methods we used in Sect. 2. In Sect. 3, we show that the morphological appearance of dust inside H II bubbles, blown by stars with $\left.\log \left(L / L_{\odot}\right) \lesssim 5.2\right)$, is often characterized by an arc-like structure emitting in the infrared (IR), and propose that these arcs are dust waves (Ochsendorf et al. 2014), where radiation pressure has stalled the dust carried along by a photo-evaporation flow of ionized gas. We test our hypothesis by performing hydrodynamical simulations of the well known H II bubble RCW 120 and present a model for studying the coupling between gas and dust, and for predicting the location of a dust wave. We discuss our findings and summarize our conclusions in Sect. 4.

\section{Observations and method}

\subsection{Surveys of the Galactic plane}

The Galactic plane has been observed at a wide range of wavelengths, allowing for a multi-wavelength study of the H II bubbles seen in the GLIMPSE survey (Churchwell et al. 2006). We explored several large-scale surveys that are publicly available online. We summarize these surveys in Table 1.

\subsection{Hydrodynamical simulations}

Hydrodynamical simulations were produced with FLASH HC (hybrid characteristics), a modified version of FLASH (Fryxell et al. 2000) that includes radiative transfer (Rijkhorst et al. 2006). FLASH is a publicly available, modular, parallel, adaptive-mesh-refinement hydrodynamical code. The transfer of ionizing radiation is carried out by the method of hybrid characteristics, which efficiently traces rays across the block-structured adaptive-mesh-refinement grid and computes the intensity of radiation at every computational cell. We adopted the on-the-spot approximation, that is, all the ionizing photons produced by recombinations to the ground state were assumed to be absorbed locally, making the radiation transfer equation more simple to solve. Since its first description by Rijkhorst et al. (2006), the HC scheme has been improved, as described in Raicevic (2010) and Verdolini (2014). The updated version of the scheme was employed in the radiative-transfer-code comparison project by Iliev et al. (2009), and tested further for a high-density medium in Verdolini (2014).

For the simulations presented in this work, we allowed the grid to refine and de-refine according to the second-order density derivative. This choice has the effect of increasing the resolution elements where the density changes on a small spatial scale. Along the edges of the $\mathrm{H}$ II region the resolution increases up to the maximum permitted, which allowed us to properly follow the evolution of the region without an excessive use of computational power.

\section{Results}

Figure 1 presents RCW 120 and RCW 82, which are two wellisolated bubbles, allowing for a detailed study of their morphology and characteristics (Zavagno et al. 2007; Pomarès et al. 2009). The two bubbles are powered by stars below the so-called weak-wind limit (i.e. $\log \left(L / L_{\odot}\right) \lesssim 5.2$ ): the ionizing source of RCW 120 is a star of spectral type O6V-O8V (Zavagno et al. 2007; Martins et al. 2010), while RCW 82 is ionized by two stars of spectral type O7V-B2V (Pomarès et al. 2009). The dust properties of both regions have been discussed in Anderson et al. (2012); their analysis, however, focused on the cold component $(10-35 \mathrm{~K})$ seen at long wavelengths $(>70 \mu \mathrm{m})$, which predominantly traces large columns of material of the dense shell. In this work, we focus on the warm dust component, seen at $24 \mu \mathrm{m}$, inside the ionized region of $\mathrm{H}$ II bubbles (see also Watson et al. 2008; Deharveng et al. 2010).

The warm dust component at $24 \mu \mathrm{m}$ is characterized by arcshaped emission that peaks close to the ionizing source and reveals that dust resides well within the borders of the swept-up shell, which is traced by $8 \mu \mathrm{m}$ emission and appears to be broken or punctured. To study the spatial distribution of gas and compare it with dust, we used high-resolution $\mathrm{H} \alpha$ emission data and complemented this with (low-resolution) radio continuum data not affected by extinction in the line of sight. These properties - an arc-shaped structure at $24 \mu \mathrm{m}$ and an incomplete shell traced at $8 \mu \mathrm{m}$ - are common properties of H II bubbles (Watson et al. 2008; Deharveng et al. 2010), see also Verdolini et al., in prep. We note that ionized gas traces the emission along the line of sight, whereas $24 \mu \mathrm{m}$ emission predominantly arises from dust heated to temperatures of $\sim 100 \mathrm{~K}$ near the star. This emission could either originate from small stochastically heated particles, often referred to as very small grains (VSGs), or big grains (BGs) in thermal equilibrium with the radiation field (e.g., Paladini et al. 2012). Whereas several authors attribute the $24 \mu \mathrm{m}$ emission inside ionized regions to the increase of VSGs compared with BGs (Paradis et al. 2011; Flagey et al. 2011), the analysis of the IR arc in IC 434 revealed that the increased heating by stellar photons from the nearby stars, $\sigma$ Ori AB, can explain the MIR emission (Ochsendorf et al. 2014). Here, we sidestep this problem and note that the emission from $24 \mu \mathrm{m}$ and $\mathrm{H} \alpha$ /radio throughout the bubble interiors shown in Fig. 1 are not spatially correlated: dust emission peaks close to the ionizing source, while the ionized gas does not follow this trend. This implies that gas and dust are (partially; see Sect. 4) decoupled inside RCW 120 and RCW 82.

We propose that H II bubbles, blown by stars with $\left.\log \left(L / L_{\odot}\right) \lesssim 5.2\right)$, are formed by thermal pressure of the gas that accompanies ionization and not by a stellar wind. The proposed scenario is depicted in Fig. 2. Using the well-studied bubble RCW 120 as reference, we performed hydrodynamical simulations to test our hypothesis.

\subsection{Hydrodynamical simulations of RCW 120}

Stars capable of creating large bubbles are thought to form inside infrared dark clouds (IRDCs), which have typical sizes of $1-3 \mathrm{pc}$, densities of $\sim 10^{4} \mathrm{~cm}^{-3}$, masses of $\sim 10^{4} \mathrm{M}_{\odot}$ (Rathborne et al. 2006) and are contained in a larger scale molecular cloud. We attempted to model the conditions inside an IRDC by constructing a computational domain containing a Bonnor-Ebert (BE) sphere of mass $\sim 7 \times 10^{5} M_{\odot}$ and radius $\sim 9 \mathrm{pc}$. The observed density profiles of cloud cores are close to those 

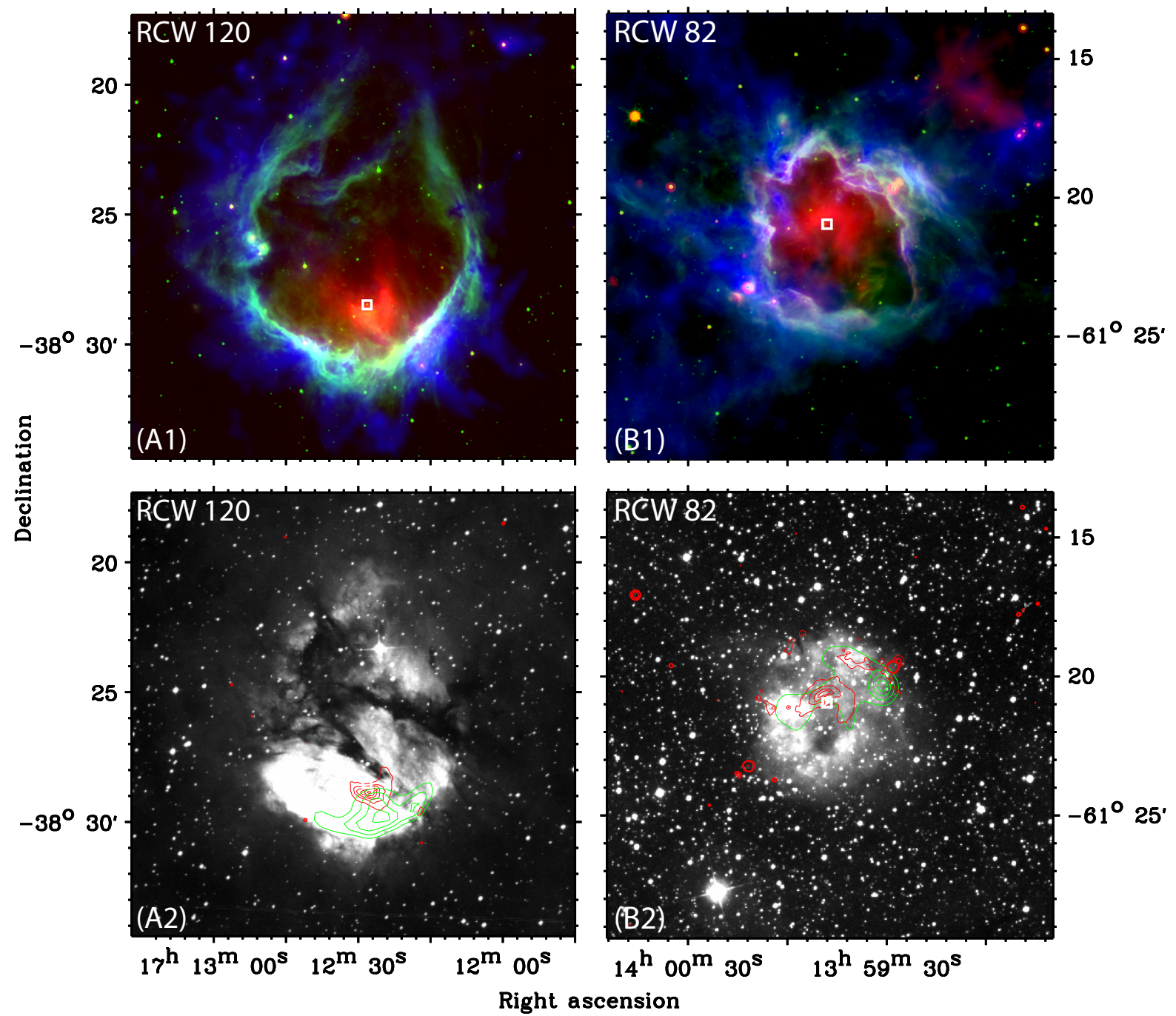

Fig. 1. In A1) H II bubble RCW 120 is ionized by a central source (white square) of spectral type O6V-O8V (Zavagno et al. 2007; Martins et al. 2010). Spitzer/IRAC observations at $8 \mu \mathrm{m}$ (green) trace PAH emission from the inner edge of the bubble, while arc-shaped $24 \mu \mathrm{m}$ emission from large $\sim 100 \mathrm{~K}$ dust grains, as seen by Spitzer/MIPS, peaks close to the central star (red). The Herschel/SPIRE $250 \mu \mathrm{m}$ observations are plotted in blue, revealing high column densities of cold $(\sim 15-30 \mathrm{~K})$ dust surrounding the bubble. Sequential star formation can occur in the swept-up material because the shells are prone to gravitational instabilities (Zavagno et al. 2007; Pomarès et al. 2009; Martins et al. 2010). The shell appears to be broken towards the top. A2) $\mathrm{H} \alpha$ SuperCOSMOS image, overlaid with contours of Spitzer/MIPS $24 \mu \mathrm{m}$ (red) and NVSS radio continuum emission at $1.4 \mathrm{GHz}$ (green), revealing the distribution of gas and dust inside the bubble. Contour levels are $\max -80 \%-60 \%-40 \%$. In B1) and B2) RCW 82 is ionized by two stars of spectral type O7V-B2V (Pomarès et al. 2009). Images B1) and B2) use the same color scheme as A1) and A2), respectively. The radio continuum data for RCW82 are taken from SUMSS at $843 \mathrm{MHz}$ with contour levels max $-80 \%-60 \%-40 \%$. In this case, the bubble appears to be broken towards the bottom.

seen in the BE model (Ballesteros-Paredes et al. 2003; Pirogov 2009) and, therefore, are often used in cloud-core simulations (e.g. Girichidis et al. 2011).

A BE sphere is an isothermal gas sphere in hydrostatic equilibrium in a pressurized medium. As initial condition, we set a density profile that follows the BE sphere solution, and did not include unnecessary calculations due to the presence of gravity. We omitted the force of gravity in our simulations because we are only interested in the interaction of the ionizing photons of the star with the surroundings, not in the formation of the star itself. We generated an initial equilibrium solution without gravity by fixing a constant pressure in the computational box and by changing the temperature accordingly. We placed the star $\sim 3 \mathrm{pc}$ offset from the center of the sphere. Dust is not included in our simulations; however, dust and gas are poorly coupled in evolved H II regions (see Sect. 4). Dust competes for ionizing photons and will shrink the HII region in size, which is captured in the uncertainty of ionizing power of the central source and will not affect our conclusions. The number of ionizing photons $Q_{0}$ is set to $3.8 \times 10^{48}$ photons s $^{-1}$ (Zavagno et al. 2007). We followed the evolution of the H II bubble during the first few Myr of expansion.

Figure 3 shows the results of a two-dimensional simulation with a maximum refinement level equal to 6 . Initially, the star creates an expanding sphere of ionized gas, surrounded by a thick shell of neutral swept-up material (Spitzer 1978). However, the density gradient of the BE sphere causes the expansion to accelerate towards low density immediately after ionization $(t<1 \mathrm{Myr})$, initiating a photo-evaporation flow inside the ionized gas. Eventually $(1<t<2 \mathrm{Myr})$, the swept-up shell reaches the edge of the sphere; gas inside the bubble is accelerated to $15-20 \mathrm{~km} \mathrm{~s}^{-1}$. After $t>3 \mathrm{Myr}$, the shell breaks open 


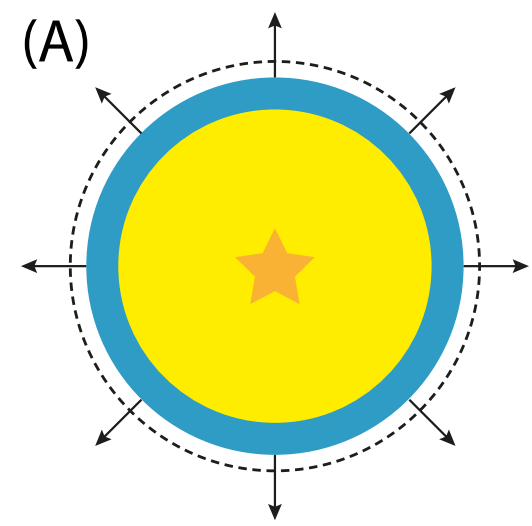

(B)

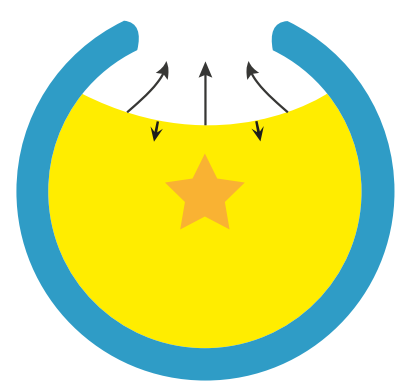

(C)

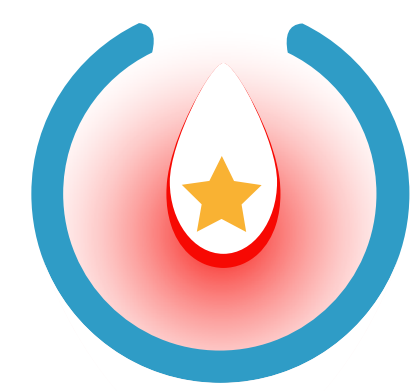

Fig. 2. A) Overpressure of the hot, ionized interior (yellow) will cause the bubble to expand inside the natal cloud, sweeping up neutral gas in a dense shell (blue) (Spitzer 1978). If the expansion is supersonic, a shock front forms on the neutral side of the shell (dashed line). B) If the ionized gas contains a density gradient and/or the bubble is punctured, a flow of ionized gas will stream towards lower density and ultimately into the surrounding ISM, relieving the bubble from its pressure (Tenorio-Tagle 1979). C) Dust is dragged along in an ionized flow, where "upstream" dust approaching the ionizing source will be heated and halted by radiation pressure, resulting in a dust wave or bow wave (Ochsendorf et al. 2014; and see Sects. 3.2 and 4), which can be traced at mid-infrared wavelengths (red).

towards the low-density region, while the ionized gas inside the $\mathrm{H}$ II region reaches velocities of about $40 \mathrm{~km} \mathrm{~s}^{-1}$. As the socalled champagne flow phase develops (Tenorio-Tagle 1979), the relative velocity between the ionized gas in the flow and the star increases (Fig. 4), creating the conditions required for the development of a dust wave (see Sects. 3.2 and 3.3). We note that the velocity of the flow, $v_{\mathrm{f}}$, depends on the starting conditions of the simulations; in particular, the offset of the star from the cloud center and the steepness of the density gradient in the parental cloud, where higher velocities are obtained within a lower density or a steeper gradient. This will be thoroughly addressed in a forthcoming paper (Verdolini et al., in prep.).

Figure 4 shows the velocity of the ionized gas and the amount of kinetic energy $\left(E_{\mathrm{k}}\right)$ transferred to the surrounding interstellar medium (ISM), calculated through a three-dimensional simulation with a maximum refinement level equal to 5 . We saved computing power by running the simulation at a lower refinement level than in the two-dimensional case shown in Fig. 3. This change in resolution does not affect the result of the run, because the energy budget of the simulation does not depend on the refinement level (Verdolini 2014; Freyer et al. 2003). We traced the amount of energy in sub- $\left(<10 \mathrm{~km} \mathrm{~s}^{-1}\right)$ and supersonic $\left(>10 \mathrm{~km} \mathrm{~s}^{-1}\right)$ components of the ionized and neutral gas, respectively. Our calculations show that initially, most of the energy is in the neutral shell of swept-up gas. As the neutral shell is accelerated, $E_{\mathrm{k}}$ is transferred into the neutral gas moving supersonically. The total energy deposited rises steadily as the expanding shell sweeps up ambient material, reaching $\sim 10^{50}$ erg over the lifetime of the star ( 10 Myr).

\subsection{Model for dusty photo-evaporation flows}

The density gradient inside the bubble shown in Fig. 3 leads to a flow of ionized gas towards lower density. The hydrodynamical simulation did not contain dust; however, in reality, dust will be contained inside the flow and be coupled to the gas through gas-grain interactions. The motion of a dust grain contained in a photo-evaporation flow can be calculated by solving a set of coupled differential equations, including the equation of motion, which balances the radiation pressure force from the star with the drag force through collisions with the gas. Here, we write the relevant equations used in this work, but for a detailed description of the physics of a dusty photo-evaporation flow we refer to Ochsendorf et al. (2014).

The equation of motion for dust is written as:

$m_{\mathrm{d}} v_{\mathrm{d}} \frac{\mathrm{d} v_{\mathrm{d}}}{\mathrm{d} r}=-\frac{\sigma_{\mathrm{d}} \bar{Q}_{\mathrm{rp}} L_{\star}}{4 \pi c r^{2}}+F_{\text {drag }}$,

where $v_{\mathrm{d}}$ is the velocity of the dust, and the first term on the righthand side represents the radiation pressure $F_{\text {rad }} ; m_{\mathrm{d}}$ is the grain mass; $r$ is the distance to the star; $L_{\star}$ and $c$ are the luminosity and the speed of light; $\sigma_{\mathrm{d}}$ and $\bar{Q}_{\mathrm{rp}}$ are the geometrical crosssection and the flux-weighted mean radiation pressure efficiency of the grain. The collisional drag force $F_{\text {drag }}$ is estimated through (Draine \& Salpeter 1979)

$F_{\text {drag }}=2 \sigma_{\mathrm{d}} k T n_{i} \frac{8 s}{3 \sqrt{\pi}}\left(1+\frac{9 \pi}{64} s_{i}^{2}\right)^{1 / 2}$,

where $k$ is the Boltzmann constant, $T$ and $n_{\mathrm{i}}$ are the temperature and number density of the gas (of species $i$ ) and $s_{i}=$ $\left(m_{i} v_{\text {drift }}^{2} / 2 k T\right)^{1 / 2}$, with $v_{\text {drift }}$ the drift velocity, which is the relative velocity of the grains with respect to the gas. The equation of motion of gas consists of a balance between momentum gained (or lost) through a pressure gradient and the momentum transfer through interactions with dust grains,

$v_{\mathrm{g}} \frac{\mathrm{d} v_{\mathrm{g}}}{\mathrm{d} r}=v_{\mathrm{g}} \frac{c_{\mathrm{s}}}{\rho_{\mathrm{g}}} \frac{\mathrm{d} \rho_{\mathrm{g}}}{\mathrm{d} r}+\frac{n_{\mathrm{d}}}{\rho_{\mathrm{g}}} F_{\mathrm{drag}}$,

where $v_{\mathrm{g}}$ and $\rho_{\mathrm{g}}$ are the velocity and density of the gas, respectively, and $c_{\mathrm{s}}$ is the local sound speed. The dust number density $n_{\mathrm{d}}$ is calculated from the MRN size distribution. The ratio $n_{\mathrm{d}} / \rho_{\mathrm{g}}$ is not constant, but will depend on $v_{\text {drift }}$ and follows from the equations of continuity.

Consider a dusty photo-evaporation flow, with initial velocity $v_{\mathrm{f}}$, approaching a star radially. The radiation pressure of the star $F_{\text {rad }}$ will act on a dust grain contained within the flow, which in this particular case will cause it to lose momentum (i.e., lower $v_{\mathrm{f}}$ ). As the dust is slowed down, it will be pushed through the gas with drift velocity $v_{\text {drift }}$, transferring momentum towards the gas through the drag force $F_{\text {drag. }}$. Eventually, the dust will be stopped at a point $r_{\min }$ ahead of the star, where the radiation pressure $F_{\text {rad }}$ balances the drag force $F_{\text {drag }}$. If at this point the amount of momentum transfer between gas and dust is insufficient for 


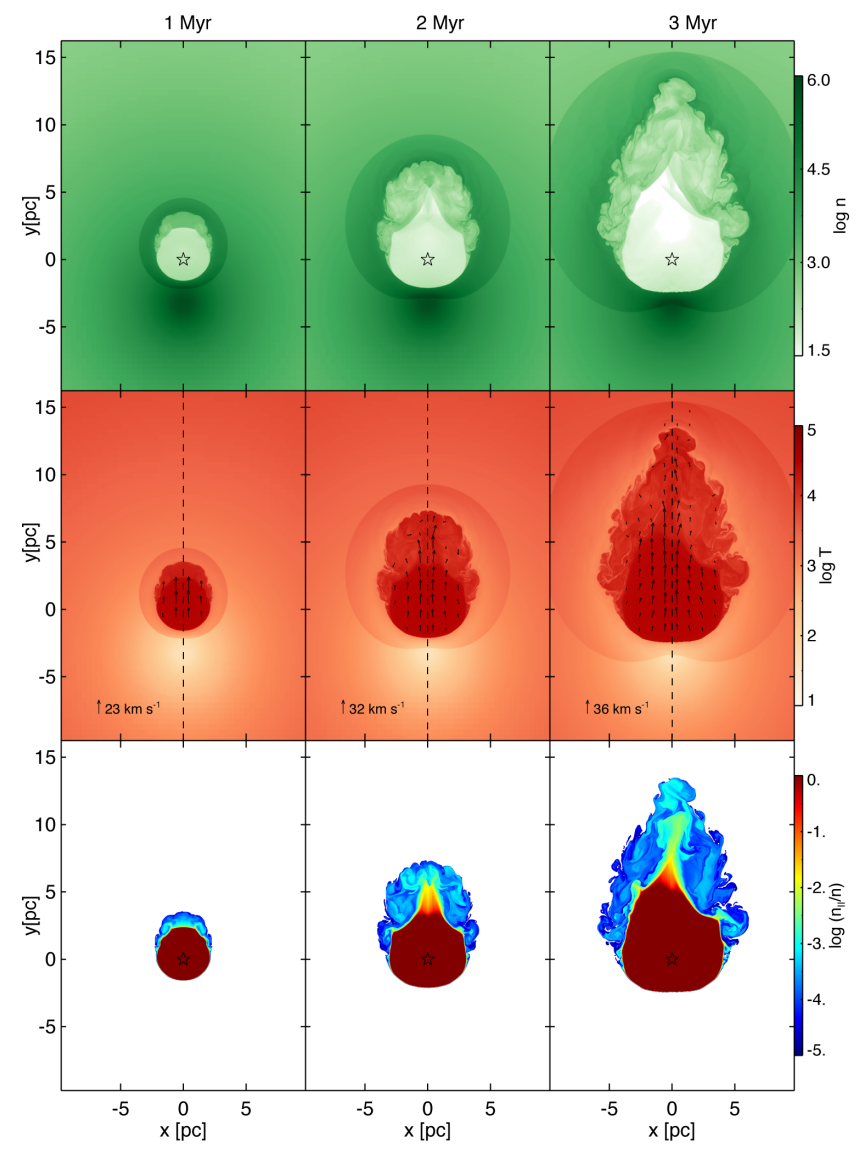

Fig. 3. Two-dimensional hydrodynamical simulation of an expanding H II region offset from the center of a Bonnor-Ebert sphere. From top to bottom rows: the logarithm of number density $n$, temperature $T$, and ionization fraction $n_{\mathrm{II}} / n$ of a slice through the $y z$-plane at the location of the star are shown. We trace the velocity of the gas in the threedimensional simulation along the dashed line (see Fig. 4A). Each column corresponds to a snapshot of the simulation at 1-3 Myr. The vectors represent the velocity field in the middle row; the legend indicates the maximum velocity.

the gas to be dynamically perturbed, the two components will decouple, resulting in an increase of dust upstream relative to the star as the gas flows along unhindered. Dust grains approaching the star with a non-zero impact parameter will be pushed around the star, resulting in an arc-shaped structure (Ochsendorf et al. 2014). The slow-down of the dust will increase the dust number density through continuity, resulting in a pile-up of dust ahead of the star. This structure has been dubbed a dust wave, the appearance of which will resemble the situation depicted in Fig. 2C. Dust waves allow us to test the properties of dust in H II regions: for example, the study of the dust wave in IC 434 implied that dust and gas in IC 434 are not coupled through Coulomb interactions, which is the reason why the plasma drag and Lorentz force are omitted in Eq. (2) (Ochsendorf et al. 2014). This unexpected result challenges our understanding of the physics of dust in H II regions, as the grains are expected to be highly charged and to be tightly coupled to the gas through Coulomb focusing.

We explored the effects of different relevant astronomical environments on the momentum transfer between gas and dust, which determines whether gas and dust will decouple to form a dust wave, or remain coupled to form a bow wave, where gas and dust flow around the star together (Ochsendorf et al. 2014). To do so, we solved the situation sketched above, where a
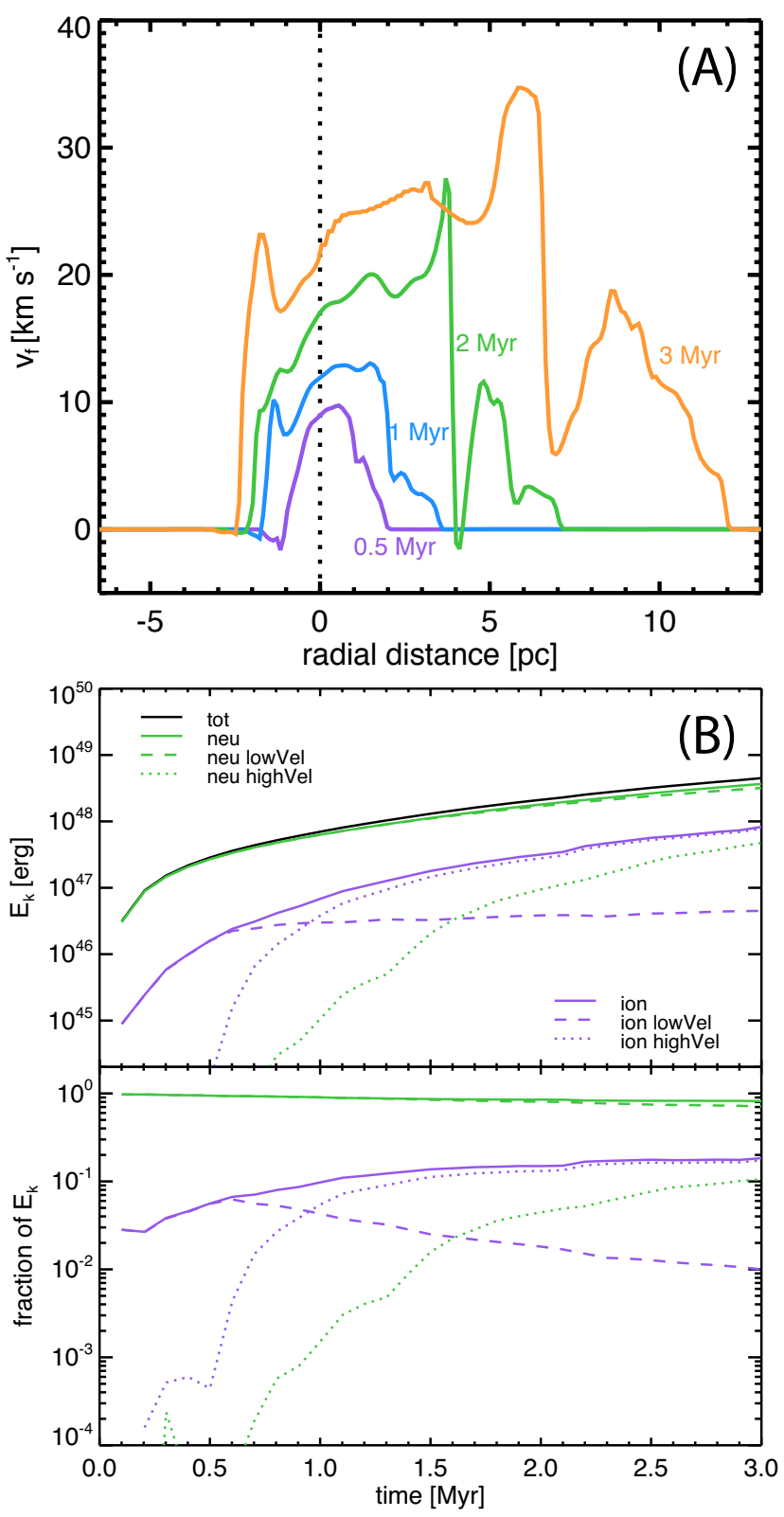

Fig. 4. A) Gas velocity along a line in the three-dimensional simulation, which runs from the star through the opening of the bubble (as exemplified in Fig. 3). Plotted are the velocity profiles at different times $t$. The star is located at the dotted line. The sharp increase of the velocity close to the ionization front at $t=1 \mathrm{Myr}$ and $t=3 \mathrm{Myr}$ are turbulent motions induced by the finite grid of the simulation. Velocities of the gas with respect to the star range from $v_{\mathrm{f}}=5$ to $v_{\mathrm{f}}=20 \mathrm{~km} \mathrm{~s}^{-1}$. B) Kinetic energy $E_{\mathrm{k}}$ contained in the three-dimensional simulation (top panel) and fractional distribution of $E_{\mathrm{k}}$ (bottom panel). We separate $E_{\mathrm{k}}$ located into the neutral gas (green), the ionized gas (purple) and their total (black line), which are further divided into the subsonic (dashed) and the supersonic (dotted) part of each component.

photo-evaporation flow approaches a star radially from the edge of the H II region, for a range of ionizing luminosities $Q_{0}$ and densities $n_{\mathrm{H}}$.

- The ionizing luminosity $Q_{0}$ determines the size of the H II region (Tielens 2005, p. 243); the Strömgren radius of the HII region is used as the starting point of the flow. Furthermore, $Q_{0}$ sets the magnitude of the radiation pressure 
force $F_{\text {rad }}$ through extrapolation of O-star parameters listed in Martins et al. (2005a).

- The density $n_{\mathrm{H}}$ regulates the collisional drag force, $F_{\text {drag }}$, as is shown in Eq. (2) (we consider collisions with hydrogen only). A higher density leads to more collisions and, consequently, a higher momentum transfer between both components.

We evaluated our model for densities in the range $10<$ $n_{\mathrm{H}}<10^{7} \mathrm{~cm}^{-3}$ and for ionizing luminosities in the range $47<$ $\log \left(Q_{0}\right)<54$ photons s ${ }^{-1}$. Through our choice of $n_{\mathrm{H}}$ and $Q_{0}$, we set up a parameter space that covers a wide variety of astronomical environments, depicted in Fig. 5. Furthermore, we calculated the solutions of our model grid assuming several constant flow velocities $v_{\mathrm{f}}$ of 5,10 , and $20 \mathrm{~km} \mathrm{~s}^{-1}$, which represent typical velocities of ionized gas at the location of the star (Fig. 4A). Note that in reality, gas will accelerate into the H II region along a pressure gradient (represented by the first term on the right-hand side of Eq. (3), and seen in Fig. 4 through increase of $v_{\mathrm{f}}$ ). Much of this acceleration will, however, occur close to the ionization front; adopting a constant velocity is a good first approximation. Our aim is to identify the physical parameters that dominate the momentum transfer between gas and dust, the bulk of which happens close to the star, where $F_{\text {rad }}$ increases and stops the dust, and we fully expect that the initial acceleration is largely irrelevant for this discussion.

\subsection{Location of dust waves and dust-gas coupling inside a photo-evaporation flow}

Figure 6A plots the location of the dust wave $r_{\text {min }}$ for $3000 \AA$ silicate grains of specific density $\rho_{\mathrm{s}}=3.5 \mathrm{~g} \mathrm{~cm}^{-3}$, which is contained in a one-dimensional flow approaching the illuminating star radially from the edge of the H II region. The size and specific density of the grains set the inertia of the incoming grains with respect to the star and enters Eq. (1) through $m_{\mathrm{d}}=\frac{4}{3} \pi a^{3} \rho_{s}$, where $a$ is the radius of the grain. We take $\bar{Q}_{\mathrm{rp}}=1$ and $n_{\mathrm{d}} / n_{\mathrm{H}}=1.8 \times 10^{-13}$ (which is the integrated number density of carbonaceous and silicate dust grains for a bin size of the MRN distribution ranging from $1000 \AA$ to $5000 \AA$, Tielens 2005 , p. 157). The results in Fig. 6A are shown for several constant flow velocities $v_{\mathrm{f}}$.

The dust waves in RCW 120 and RCW 82 are observed at 0.23 and $0.41 \mathrm{pc}$ (Zavagno et al. 2007; Pomarès et al. 2009), and are well reproduced with a flow speed of $v_{\mathrm{f}} \sim 10-20 \mathrm{~km} \mathrm{~s}^{-1}$, which is reached in our hydrodynamical simulation after $t>$ 1 Myr (Sect. 3.1). For comparison, RCW 120 is thought to be $\sim 2.5$ Myr old (Martins et al. 2010). The exact position of $r_{\text {min }}$ will depend on the radiation pressure on the one hand and the flow parameters (i.e., the velocity $v_{\mathrm{f}}$ and density $n_{\mathrm{H}}$ of the flow) on the other hand. Figure 6A shows that the dependence of $r_{\min }$ on $v_{\mathrm{f}}$ is weak. In contrast, $v_{\mathrm{f}}$ has a strong effect on the momentum transfer from the dust to the gas, as described below.

The amount of momentum transferred from the dust to the gas depends on the gas density $n_{\mathrm{H}}$ and on the flow velocity $v_{\mathrm{f}}$. The ambient number density $n_{\mathrm{H}}$ sets the number of collisions between gas and dust. At high $n_{\mathrm{H}}$, the collisions between gas and dust increase in frequency and the amount of momentum transfer to the gas is larger. The velocity of the flow $v_{\mathrm{f}}$ sets the time $t$ that it takes for the flow to travel the distance from the edge of the Strömgren sphere towards the central source. Furthermore, $t$ determines how much momentum can be transferred to the gas over time. Our calculations show that even though fast evaporation flows eventually lead to a higher drift velocity of the grains $v_{\text {drift }}$

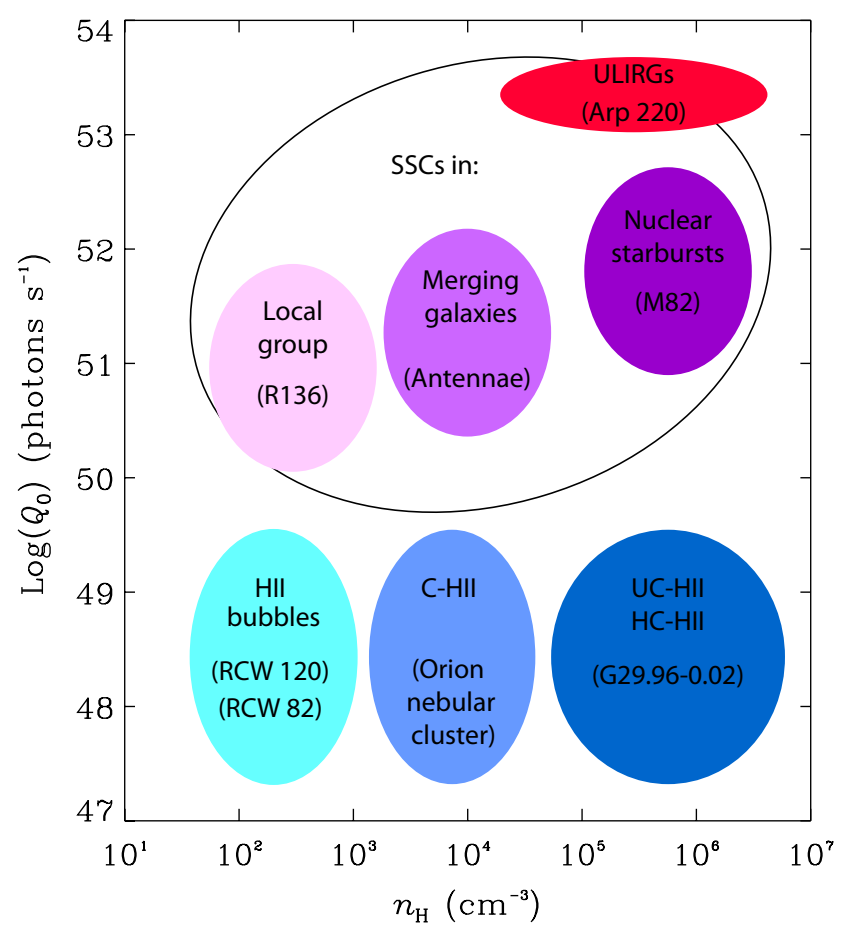

Fig. 5. Astronomical environments covered by the parameter space through our choice of the ionizing luminosity $Q_{0}$ and density $n_{\mathrm{H}}$. Among the environments shown are ultra-compact and hyper-compact HII regions (UC-H II/HC-HII), compact H II regions (C- $\mathrm{HII}$ ), and evolved H II bubbles, as discussed in this work. We also show super star clusters (SSCs) in the Local Group, merging galaxies, starburst galaxies, and ultra luminous infrared galaxies (ULIRGs). Prototypical candidates of each class are denoted between brackets (for references, see Fig. 6).

through the gas (i.e., a higher $F_{\text {drag }}$ ) and therefore more momentum transfer between the grains to the gas per unit time, the short timescale for a fast photo-evaporation flow to reach $r_{\text {min }}$ causes the total momentum transferred to decrease compared with a slow-moving flow of similar density $n_{\mathrm{H}}$. To summarize, momentum transfer between gas and dust is most efficient in a slow-moving, high-density photo-evaporation flow.

We have expressed the amount of momentum transfer through the coupling strength parameter $C$, which is a measure of the efficiency of momentum transfer between gas and dust and defined as $C=1-p_{1} / p_{0}$, where $p_{0}$ and $p_{1}$ are the initial momentum of the gas (set by $v_{\mathrm{f}}$ ) and the momentum of the gas at the position where dust reaches $r_{\text {min }}$, respectively. Note that in our model, the dust number density per unit mass of gas $\left(=n_{\mathrm{d}} / \rho_{\mathrm{g}}\right)$ is determined through the relative velocity of the gas and dust through the continuity equation and, as such, is not defined at $r_{\text {min }}$ where the dust velocity $v_{\mathrm{d}}$ reaches zero. This is a peculiarity of the streamline approaching the star radially: in reality, dust along this streamline will acquire momentum in a random direction because of Brownian motion and flow past the star before the dust is stopped completely. Therefore, we opted to evaluate the momentum transfer up to the point where the dust reaches a velocity to the expected Brownian motion velocity $\left.v_{\mathrm{B}}=\sqrt{8 k T / \pi m_{\mathrm{d}}}=2.6 \times 10^{-4} \mathrm{~km} \mathrm{~s}^{-1}\right)$.

If $C=1$, dust and gas remain perfectly coupled inside a photo-evaporation flow, and gas will be stopped along with dust. Observationally, a high value of $C$ will express itself in a low contrast between gas and dust and both components would appear to be spatially correlated (a bow wave), similar to the 

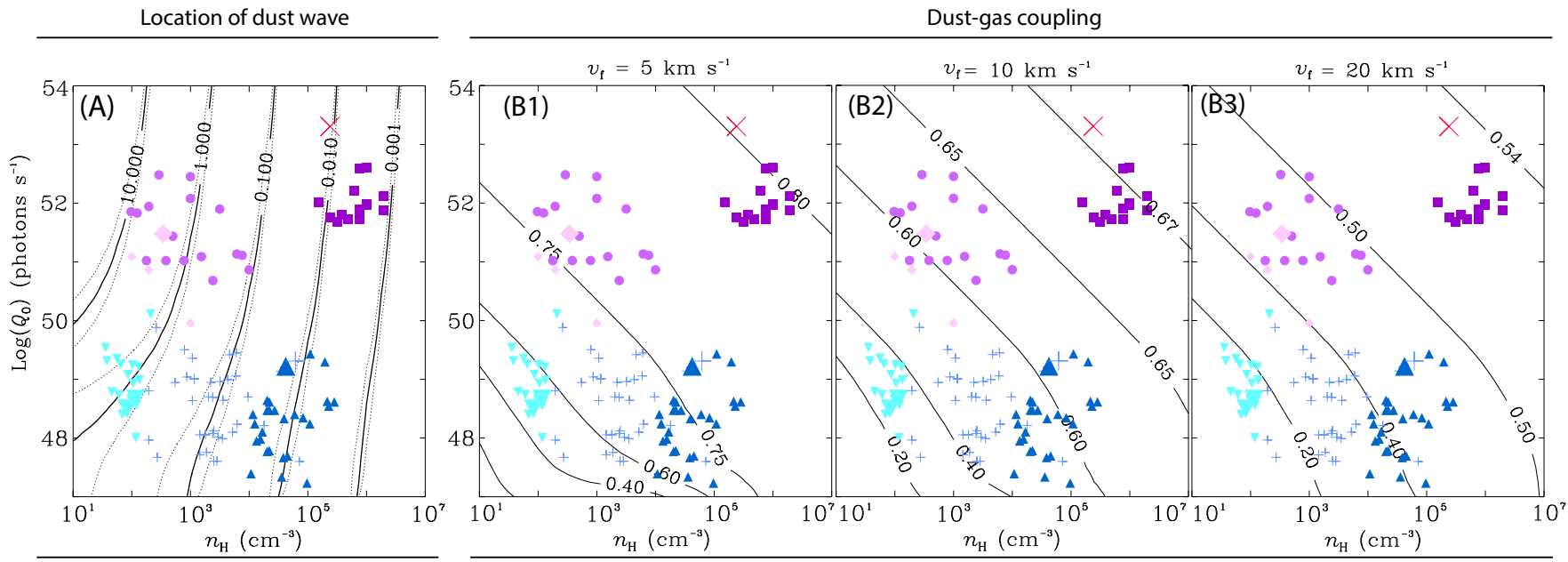

Fig. 6. A) Location of a dust wave $r_{\min }$ (black contours) in pc evaluated for a grid of ionizing source luminosities $Q_{0}$ and ambient densities $n_{\mathrm{H}}$ for $v_{\mathrm{f}}=10 \mathrm{~km} \mathrm{~s}^{-1}$. The dotted contours represent $r_{\text {min }}$ calculated with $v_{\mathrm{f}}=5 \mathrm{~km} \mathrm{~s}^{-1}$ and $20 \mathrm{~km} \mathrm{~s}^{-1}$ (a lower flow velocity will move the contours to the right in this figure). Overplotted are different astronomical objects, located in regimes drawn in Fig. 5: UC- H II /HC- H II; $\boldsymbol{\Delta}$ (Wood \& Churchwell 1989), C-H II; + (Garay et al. 1993) and evolved H II bubbles; $\nabla$ (Paladini et al. 2012). We also show SSCs in the Local Group; (compiled from Turner 2009), merging galaxies; (Gilbert \& Graham 2007), starburst galaxies; (McCrady \& Graham 2007), and ULIRGs; $\times$ (Anantharamaiah et al. 2000). Larger symbols correspond to prototypical candidates of a class denoted between brackets Fig. 5. B1), B2), and B3) coupling strength $C$ (black contours), evaluated for a flow velocity $v_{\mathrm{f}}=5 \mathrm{~km} \mathrm{~s}^{-1}, 10 \mathrm{~km} \mathrm{~s}^{-1}$, and $20 \mathrm{~km} \mathrm{~s}^{-1}$.

appearance of a stellar-wind bow shock (van Buren et al. 1990; Kaper et al. 1997). When $C<1$, dust and gas decouple and a higher contrast between dust and gas distribution will be seen in sources with decreasing $C$ (a dust wave). In panels B1, B2, and B3 of Fig. 6, we plot $C$ at different flow velocities $v_{\mathrm{f}}$. In all cases, $C$ is lowest in evolved bubble HII regions, which is the main reason that a high contrast between the distribution of gas and dust can be seen inside these sources. However, $v_{\mathrm{f}}$ has a significant impact on the magnitude of momentum transfer: for example, at $v_{\mathrm{f}}=5 \mathrm{~km} \mathrm{~s}^{-1}, C \sim 0.5$ for RCW 120 and RCW 82 . In contrast, at $v_{\mathrm{f}}=20 \mathrm{~km} \mathrm{~s}^{-1}, C<0.2$. Again, gas and dust couple relatively well in slower photo-evaporating flows, which could lead to the appearance of a bow wave (Ochsendorf et al. 2014), where dust and gas move around the star together.

\section{Discussion and conclusion}

The IR emission inside H II bubbles is often characterized by arc-structures, as exemplified in RCW 120 and RCW 82. We have argued that these structures can be explained as dust waves induced by photo-evaporating flows inside H II bubbles, which are initiated either by a density gradient inside the bubble or an opening in the bubble shell. The escaping gas will eventually be replaced by a flow of ionized gas evaporating from the inner wall of the swept-up shell. This mechanism provides a natural explanation for the presence and morphology of dust emission seen in the interior of H II bubbles (Deharveng et al. 2010; Martins et al. 2010). Our simulations confirm that an O8 V star can form a bubble of several pc across in typical IRDC conditions without a stellar wind. The bubble is inflated by overpressure of the ionized gas and reveals a similar structure as in RCW 120 and RCW 82, where an ionized gas volume surrounded by a dense shell opens up to one side in order to create a fast champagne flow.

In this work, we investigated bubble H II formation in the absence of a stellar wind. As outlined in Sect. 1, recent observations have challenged the WBB model (Weaver et al. 1977), given the weak-wind strengths measured for stars with $\log \left(L / L_{\odot}\right) \lesssim 5.2$, the difficulty in detecting diffuse X-rays around main-sequence stars, and the presence and morphology of dust inside H II bubbles. We do not imply that stellar winds are intrinsically absent in these bubbles; the scenario presented here offers an alternative scenario for the formation and evolution of H II bubbles seen by Spitzer and Herschel because it circumvents the previously mentioned problems. The bubble sample of Beaumont \& Williams (2010) indicates that the majority of H II bubbles are indeed blown by stars below the weakwind limit (i.e. $\log \left(L / L_{\odot}\right) \lesssim 5$.2), even after correcting the distance toward the bubbles, which were shown by Anderson \& Bania (2009) to be systematically higher than the values reported by Beaumont \& Williams (2010). However, sources approaching luminosities of $\log \left(L / L_{\odot}\right) \sim 5.2$ may have a stellar wind contributing to the morphology of gas and dust (see Draine 2011). Moreover, several large bubbles powered by a cluster of stars, with luminosities near to or possibly exceeding the weakwind limit, also exhibit IR arcs in their interior, for instance, the W5 region (Koenig et al. 2008). In this case, dust and gas seem to follow a similar morphology inside the bubble (Deharveng et al. 2012), which either is the signature of a bow wave or the effect of a stellar wind, as it is unlikely that both the dust and gas will penetrate in the inner region of a WBB dominated by the freeflowing wind (Weaver et al. 1977), and will flow around this region together. More simulations to study gaseous flows inside WBBs will help in understanding the evolution and morphology of bubbles around sources that approach or exceed the weakwind limit. Nevertheless, dust waves provide direct evidence for the importance of bubble champagne flows, irrespective of the dominant source of expansion (i.e. thermal pressure or stellar winds).

Dust waves require an appreciable flow speed $\left(v_{\mathrm{f}}>10 \mathrm{~km} \mathrm{~s}^{-1}\right)$ of the gas near the star for the dust to significantly decouple from the gas. This is reproduced in our models by placing the star offset from the center of a BonnorEbert sphere, whose density gradient leads to a significant acceleration of the gas after ionization of the cloud material. In addition to the acceleration that is needed to segregate the gas 
and dust to create a dust wave, the density gradient in the cloud also leads to (narrow) openings through which the gas is vented into the ISM, which is characteristic for many bubbles such as RCW 120 and RCW 82 (Zavagno et al. 2007; Pomarès et al. 2009; Deharveng et al. 2010).

We note that the evolution of interstellar bubbles can be affected by several other mechanisms, such as the motion of the ionizing source, which leads to a cometary shape of the bubble in the direction of movement (Wood \& Churchwell 1989), and an external magnetic field, which adds anisotropic pressure to the bubble (Bisnovatyi-Kogan \& Silich 1995), possibly leading to elongated shapes aligned along the Galactic field lines (Pavel \& Clemens 2012). This does not seem to apply for RCW 120 and RCW 82, given the near-spherical appearance of the two regions. In addition, we note that grain charging inside $\mathrm{H}$ II regions is not fully understood, and motions of dust grains in IC 434 does not seem to be influenced by Coulomb interaction with the plasma (Ochsendorf et al. 2014). For IC 434, the morphological appearance of the photo-evaporation flow indicates a free flow of the gas and a magnetic field, if present, would then most likely be oriented perpendicular to the cloud surface. Again, it is unclear whether a similar explanation applies to the bubbles studied in this paper.

Large turbulent instabilities arise once the ionized flow inside the bubble clashes with the dense shell; the signatures of Kevin-Helmholtz and Rayleigh-Taylor instabilities are clearly seen in Fig. 3. However, this does not affect the formation of the dust wave contained within the ionized part of the bubble, because the flow energy of the ionized gas is much higher than the turbulence energy, resulting in a smooth, laminar flow of ionized gas in the interior of the bubble as exemplified in Fig. 3. Moreover, gas and dust are only weakly coupled inside evolved H II regions (see Fig. 6), which minimizes the influence of turbulence on the motion of dust inside a photo-evaporation flow.

Our models predict that the flow becomes highly supersonic across the bubble, channeling its contents into the surrounding ISM. For the most common type II supernova progenitor (late O/early B-stars with a main-sequence lifetime of 10 Myr), some $10^{50}$ erg of kinetic energy can be deposited into the ISM. This is similar to the typical supernova energy $\left(\sim 10^{51} \mathrm{erg}\right.$, of which $\sim 10 \%$ is transferred to kinetic energy of the interstellar gas Veilleux et al. 2005). Moreover, while on a large scale the structure of the ISM is dominated by the walls and chimneys associated with superbubbles created by supernovae breaking into the Galactic halo, Spitzer and Herschel surveys demonstrate that on smaller scales the structure of the ISM is controlled by the $\mathrm{H}$ II bubbles and photo-evaporation flows discussed here.

The momentum transfer between dust and gas in evolved H II bubbles such as RCW 120 and RCW 82 is insufficient for the gas to be dynamically perturbed. This makes these objects ideal candidates for observing dust waves and for studying the properties of dust in HII regions and their dynamical interaction with stellar radiation and gas. However, the velocity of the flow is an important factor: at low velocities, gas remains coupled more efficiently and can form a bow wave. This situation will resemble a stellar wind bow-shock configuration (van Buren et al. 1990; Kaper et al. 1997), where gas and dust peak at a position upstream and produce a drop in emission measure downstream with respect to the star. In regions of high density such as the (ultra)-compact H II regions, the collision rate between dust and gas increases and the coupling between the two components tightens. Dust and gas also couple well in SSC environments such as R136 in the 30 Dor region and the nuclear starburst in M82. In particular in the latter, dust and gas show similar morphologies near the SSCs that are launching a galactic wind (Gandhi et al. 2011). Radiation pressure is expected to play a key role in the gas and dust dynamics in ULIRGs such as Arp 220. These immense star-forming galaxies will be most efficient in driving gas dynamics through coupling with dust, thereby limiting the efficiency of star formation (Andrews \& Thompson 2011).

Recent studies (Krumholz \& Matzner 2009; Draine 2011; Silich \& Tenorio-Tagle 2013) have explored the importance of radiation pressure for the dynamics of gas around young star clusters. This mechanism is poorly understood, mainly because of our limited understanding of the interplay between radiation pressure, dust, and gas. The study of photo-evaporation flows and dust waves provides us with a unique laboratory to directly study the momentum coupling of stellar radiation and the surrounding medium, which largely proceeds through the dust and is crucial for the implementation in current state-of-the-art models on star and galaxy formation and evolution throughout the history of the Universe.

Acknowledgements. Studies of interstellar dust and chemistry at Leiden Observatory are supported through advanced ERC grant 246976 from the European Research Council, through a grant by the Dutch Science Agency, NWO, as part of the Dutch Astrochemistry Network, and through the Spinoza premie from NWO. N.L.J.C. acknowledges support from the Belgian Federal Science Policy Office via the PRODEX Programme of ESA. The software used in this work was in part developed by the DOE NNSA-ASC OASCR Flash Center at the University of Chicago.

\section{References}

Anantharamaiah, K. R., Viallefond, F., Mohan, N. R., Goss, W. M., \& Zhao, J. H. 2000, ApJ, 537, 613

Anderson, L. D., \& Bania, T. M. 2009, ApJ, 690, 706

Anderson, L. D., Zavagno, A., Deharveng, L., et al. 2012, A\&A, 542, A10

Andrews, B. H., \& Thompson, T. A. 2011, ApJ, 727, 97

Arthur, S. J. 2012, MNRAS, 421, 1283

Ballesteros-Paredes, J., Klessen, R. S., \& Vázquez-Semadeni, E. 2003, ApJ, 592, 188

Beaumont, C. N., \& Williams, J. P. 2010, ApJ, 709, 791

Benjamin, R. A., Churchwell, E., Babler, B. L., et al. 2003, PASP, 115, 953

Bisnovatyi-Kogan, G. S., \& Silich, S. A. 1995, Rev. Mod. Phys., 67, 661

Bock, D. C.-J., Large, M. I., \& Sadler, E. M. 1999, AJ, 117, 1578

Carey, S. J., Noriega-Crespo, A., Mizuno, D. R., et al. 2009, PASP, 121, 76

Chu, Y.-H., \& Mac Low, M.-M. 1990, ApJ, 365, 510

Churchwell, E., Povich, M. S., Allen, D., et al. 2006, ApJ, 649, 759

Condon, J. J., Cotton, W. D., Greisen, E. W., et al. 1998, AJ, 115, 1693

Dale, J. E., Ngoumou, J., Ercolano, B., \& Bonnell, I. A. 2013, MNRAS, 436, 3430

Deharveng, L., Schuller, F., Anderson, L. D., et al. 2010, A\&A, 523, A6

Deharveng, L., Zavagno, A., Anderson, L. D., et al. 2012, A\&A, 546, A74

Draine, B. T. 2011, ApJ, 732, 100

Draine, B. T., \& Salpeter, E. E. 1979, ApJ, 231, 77

Everett, J. E., \& Churchwell, E. 2010, ApJ, 713, 592

Flagey, N., Boulanger, F., Noriega-Crespo, A., et al. 2011, A\&A, 531, A51

Freyer, T., Hensler, G., \& Yorke, H. W. 2003, ApJ, 594, 888

Fryxell, B., Olson, K., Ricker, P., et al. 2000, ApJS, 131, 273

Gandhi, P., Isobe, N., Birkinshaw, M., et al. 2011, PASJ, 63, 505

Garay, G., Rodriguez, L. F., Moran, J. M., \& Churchwell, E. 1993, ApJ, 418, 368

Gilbert, A. M., \& Graham, J. R. 2007, ApJ, 668, 168

Girichidis, P., Federrath, C., Banerjee, R., \& Klessen, R. S. 2011, MNRAS, 413, 2741

Harper-Clark, E., \& Murray, N. 2009, ApJ, 693, 1696

Huenemoerder, D. P., Oskinova, L. M., Ignace, R., et al. 2012, ApJ, 756, L34

Iliev, I. T., Whalen, D., Mellema, G., et al. 2009, MNRAS, 400, 1283

Kaper, L., van Loon, J. T., Augusteijn, T., et al. 1997, ApJ, 475, L37

Koenig, X. P., Allen, L. E., Gutermuth, R. A., et al. 2008, ApJ, 688, 1142

Krumholz, M. R., \& Matzner, C. D. 2009, ApJ, 703, 1352

Marcolino, W. L. F., Bouret, J.-C., Martins, F., et al. 2009, A\&A, 498, 837

Martins, F., Schaerer, D., \& Hillier, D. J. 2005a, A\&A, 436, 1049

Martins, F., Schaerer, D., Hillier, D. J., et al. 2005b, A\&A, 441, 735 
B. B. Ochsendorf et al.: Radiation-pressure-driven dust waves inside bursting interstellar bubbles

Martins, F., Pomarès, M., Deharveng, L., Zavagno, A., \& Bouret, J. C. 2010, A\&A, 510, A32

McCrady, N., \& Graham, J. R. 2007, ApJ, 663, 844

Molinari, S., Swinyard, B., Bally, J., et al. 2010, PASP, 122, 314

Najarro, F., Hanson, M. M., \& Puls, J. 2011, A\&A, 535, A32

Ochsendorf, B. B., Cox, N. L. J., Krijt, S., et al. 2014, A\&A, 563, A65

Paladini, R., Umana, G., Veneziani, M., et al. 2012, ApJ, 760, 149

Paradis, D., Paladini, R., Noriega-Crespo, A., et al. 2011, ApJ, 735, 6

Parker, Q. A., \& Phillipps, S. 1998, PASA, 15, 28

Pavel, M. D., \& Clemens, D. P. 2012, ApJ, 760, 150

Pirogov, L. E. 2009, Astron. Rep., 53, 1127

Pomarès, M., Zavagno, A., Deharveng, L., et al. 2009, A\&A, 494, 987

Puls, J., Vink, J. S., \& Najarro, F. 2008, A\&ARv, 16, 209

Raga, A. C., Cantó, J., \& Rodríguez, L. F. 2012, Rev. Mexicana Astron. Astrofis., 48,199

Raicevic, M. 2010, Ph.D. Thesis, Durham University, UK

Rathborne, J. M., Jackson, J. M., \& Simon, R. 2006, ApJ, 641, 389

Rijkhorst, E.-J., Plewa, T., Dubey, A., \& Mellema, G. 2006, A\&A, 452, 907

Silich, S., \& Tenorio-Tagle, G. 2013, ApJ, 765, 43

Simpson, R. J., Povich, M. S., Kendrew, S., et al. 2012, MNRAS, 424, 2442
Spitzer, Jr., L. 1978, J. Roy. Astron. Soc. Canada, 72, 349

Tenorio-Tagle, G. 1979, A\&A, 71, 59

Tielens, A. G. G. M. 2005, The Physics and Chemistry of the Interstellar Medium (Cambridge University Press)

Toalá, J. A., \& Guerrero, M. A. 2013, A\&A, 559, A52

Toalá, J. A., Guerrero, M. A., Chu, Y.-H., et al. 2012, ApJ, 755, 77

Townsley, L. K., Feigelson, E. D., Montmerle, T., et al. 2003, ApJ, 593, 874

Turner, J. L. 2009, in Astrophysics in the Next Decade, Astrophys. Space Sci. Proc., eds. H. A. Thronson, M. Stiavelli, \& A. Tielens, 215

van Buren, D., Mac Low, M.-M., Wood, D. O. S., \& Churchwell, E. 1990, ApJ, 353,570

Veilleux, S., Cecil, G., \& Bland-Hawthorn, J. 2005, ARA\&A, 43, 769

Verdolini, S. 2014, Ph.D. Thesis, Leiden Observatory, The Netherlands

Walch, S., Whitworth, A. P., Bisbas, T. G., Wünsch, R., \& Hubber, D. A. 2013, MNRAS, 435, 917

Watson, C., Povich, M. S., Churchwell, E. B., et al. 2008, ApJ, 681, 1341

Weaver, R., McCray, R., Castor, J., Shapiro, P., \& Moore, R. 1977, ApJ, 218, 377

Wood, D. O. S., \& Churchwell, E. 1989, ApJS, 69, 831

Zavagno, A., Pomarès, M., Deharveng, L., et al. 2007, A\&A, 472, 835 